\title{
Special issue of selected papers from visualization and data analysis 2011
}

To appear in: Information Visualization Journal

David L. Kao, NASA Ames Research Center, Moffett Field, California, USA

Pak Chung Wong, Pacific Northwest National Laboratory, Richland, Washington, USA

\section{Editorial Introduction}

This special issue features the best papers that were selected from the $18^{\text {th }}$ SPIE Conference on Visualization and Data Analysis (VDA 2011). This annual conference is a major international forum for researchers and practitioners interested in data visualization and analytics research, development, and applications. VDA 2011 was held in the City of Burlingame outside San Francisco, CA, January 23-27, 2011. VDA 2011 received 42 highquality submissions from around the world. Twenty-four papers were selected for full conference papers. The top five papers have been expanded and reviewed for this special issue. Below is a synopsis of these papers:

"Visualizing Explicit and Implicit Relations of Complex Information Spaces" by Doerk, Carpendale, and Williamson. Many datasets include both explicit and implicit relations, which are often difficult to represent and visualize. In this paper, the authors describe a new method for integrating the visualization of explicit and implicit data relations using EdgeMaps. Explicit relations are specific connections between entities already present in a given dataset, while implicit relations are derived from multidimensional data based on shared properties and similarity measures. Doerk and his coauthors applied their technique to data sets from three case studies: philosophers, painters, and musicians.

"A Web-enabled Visualization Toolkit for Geovisual Analytics" by Ho van, Lundblad, Åström, and Jern. In the past decades, we had seen a growing trend in web-enabled applications. Ho van and his coauthors introduce a framework and class library called GAV Flash, which supports customized web-enabled applications for geovisual analytics tasks. GAV Flash, which is implemented in Adobe's ActionScript, provides a collection of interactive geo- and information visualization representations for exploring highdimensional spaces that are extended with motion behavior. Ho van's toolkit is not only for interactive visualization but also supports storytelling around visual analytics.

"Visualization and Multivariate Clustering of Scattered Moment Tensors" by Obermaier, Billen, Joy, Hagan, and Hering-Bertram. Tensors play an important role in encoding physical simulation output or measurement data since they are able to represent highdimensional geometric processes such as diffusion and deformation. Tensors from applications such as fluid flows and medical imaging are widely known. Yet, tensors from the seismic earthquake measurements in geosciences are less known. Moment tensors are indefinite tensors derived from seismic measurements and encode important information 
about earthquakes and surface displacement in the earth's mantle. Obermaier and his coauthors propose visualization of the tensor field at different scales based on multivariate tensor clustering. They present the visualization of moment tensor data from earthquakes in Chile and Tonga.

"Interactive Isosurfaces with Quadratic $\mathrm{C}^{1}$ Splines on Truncated Octahedral Partitions" by Kalbe, Marinc, Rhein, and Goesele. In many applications, it is sometimes necessary to construct a continuous function based on some discrete data points. Kalbe and his coauthors propose a local approximation method for quadratic $\mathrm{C}^{1}$ splines on uniform tetrahedral partitions to produce a globally smooth function. They present visualization results using these splines, which are also well-suited for GPU-based, interactive highquality visualization of isosurfaces from discrete data.

"Visual Exploration of Frequent Patterns in Multivariate Time Series" by Hao, Dayal, Marwah, Keim, and Janetzko. In many real-world problems, there are often frequently occurring patterns. Furthermore, many problems are associated with a time series. Hao et al. propose an event encoding and pattern discovery pattern to detect frequently occurring patterns, also called motifs. A large time series can easily contain hundreds of motifs and the ability to perform interactive analysis and exploration is crucial. Hao et al. introduce four novel techniques to analyze motifs in large time series: (1) motif layout, using colored rectangles for visualizing the occurrences and hierarchical relationships of motifs; (2) motif distortion, enlarging or shrinking motifs for visualizing them more clearly; (3) motif merging, combining a number of identical adjacent motif instances to simplify the display; and (4) pattern preserving prediction, using a pattern preserving smoothing and prediction algorithm to provide a reliable prediction for seasonal data.

We hope you will enjoy reading these papers as we have.

\section{Acknowledgment}

We would like to thank our authors for their contributions. We also would like to thank the reviewers and the VDA program committee for their helpful and informative comments and contributions. We also appreciate the generous encouragement from Information Visualization by giving us the valuable opportunity to publish the special issue. 